\title{
A NOTE ON QUANTUM PROBABILITY SPACES
}

\author{
TIBOR NEUBRUNN
}

Abstract. In general the collection of sets closed with respect to countable disjoint unions and with respect to the complementation, generated by a given collection $A$ does not coincide with the $\sigma$-field generated by $A$. In the present paper two necessary and sufficient conditions for the equality of the last mentioned systems are given. The coincidence of the above systems in cases when $A$ is the collection of all open sets in a topological space is obtained as a corollary.

1. Introduction. In his paper [1] S. P. Gudder considered quantum probability spaces introduced by $P$. Suppes in [2]. This note contains a new result concerning these spaces. In one of the corollaries the positive answer to the question, whether in a topological space the $\sigma$-class $C$ generated by the class of all open sets coincides with the Borel field, is given. The method by which the result is obtained is a standard one closely related e.g. to that used by Sierpinski in [3] while examining the abstract Borel sets.

2. Notations and notions. The notion of the $\sigma$-class $C$ is used in the same sense as in [1], i.e. $C$ is a collection of subsets of a given nonempty set $\Omega$ containing $\Omega$, closed both under the forming of countable disjoint unions and complementations. The notion of the $\sigma$-field has the same meaning as in [1] and coincides with the notion of the $\sigma$ algebra as defined in [4].

3. Results. The proof of the following simple lemma is contained in [1] and therefore will be omitted.

Lemma. Let $C$ be a $\sigma$-class of subsets of $\Omega$.

(i) If $a, b \in C, a \subset b$, then $b \cap a^{\prime} \in C$.

(ii) If $a_{1}, a_{2}, \cdots \in C$, and $a_{1} \supset a_{2} \supset \cdots$, then $\bigcap_{i} a_{i} \in C$.

As it is known, the $\sigma$-class $C$ generated by a collection $A$ of sets does not coincide in general with the $\sigma$-field generated by $A$. The following theorem clarifies the relation between the above systems.

Theorem. Let $A$ be a collection of subsets of $\Omega$. Let $C$ be the $\sigma$-class

Received by the editors October 17, 1969.

AMS Subject Classifications. Primary 2810; Secondary 6005.

Key Words and Phrases. Quantum probability space, abstract Borel sets, topological space. 
generated by $A$. Either of the following conditions is necessary and sufficient for $C$ to be a $\sigma$-field.

(iii) If $a, b \in A$, then $a-b \in C$.

(iv) If $a, b \in A$, then $a \cap b \in C$.

Proof. The necessity of both the conditions is trivial. Let us prove the sufficiency of (iii). For this it is sufficient to prove that $a-b \in C$ for any $a, b \in C$. Let $a \in A$ be fixed. Denote by $\phi(a)$ the collection of all those $l \in C$ for which $a-l \in C$. Evidently $\phi(a) \supset A$ according to (iii). We shall prove that $\phi(a)$ is a $\sigma$-class. Let $l \in \phi(a)$. We have $a-(\Omega-l)=a \cap l$, hence to prove that $\Omega-l$ belongs to $\phi(a)$ it suffices to prove $a \cap l \in C$. But $a \cap l=a-(a-l)$. Since $a-l \subset a, a-l \in C$ we have $a \cap l \in C$ by (i) of the lemma. Thus $\Omega-l \in \phi(a)$.

Now we shall prove that $\phi(a)$ is closed under the forming of countable disjoint unions. First of all the closedness under the union of two, and hence of any finite number of mutually disjoint sets will be proved. Thus let $l \in \phi(a), f \in \phi(a), l \cap f=\varnothing$. We have $a-(l \cup f)$ $=(a-l)-a \cap f$. But $a \in A, f \in \phi(a)$ implies $a \cap f \in C$ as we have seen above. Since $a-l \in C, a \cap f \in C$ and $a \cap f \subset a-l$ we have again by (i) of the lemma $(a-l)-a \cap f=a-(l \cup f) \in C$. This means $l \cup f \in \phi(a)$. Now let $\left\{l_{n}\right\}$ be a sequence of mutually disjoint sets, where $l_{n} \in \phi(a)$ for $n=1,2, \cdots$. Putting $f_{k}=l_{1} \cup l_{2} \cup \ldots \cup l_{k}$ for $k=1,2, \cdots$, we have $f_{k} \in \phi(a)$, hence $a-f_{k} \in C$. Evidently $a-f_{k} \supset a-f_{k+1}$. By (ii) of lemma $\bigcap_{k}\left(a-f_{k}\right) \in C$. But $\bigcap_{k}\left(a-f_{k}\right)=a-\bigcup_{n} l_{n}$, hence $\bigcup l_{n} \in \phi(a)$. Thus $\phi(a)$ is a $\sigma$-class containing $A$. Consequently $\phi(a) \supset C$. Since evidently $\phi(a) \subset C$, we have $\phi(a)=C$. This being true for any $a \in A$, we have $a-l \in C$ for any $a \in A$ and any $l \in C$. Now let $\phi$ be the collection of all $a \in C$ such that $a-b \in C$ for any $b \in C$. According to what was proved, $\phi$ contains $A$. If $\left\{a_{n}\right\}$ is a sequence of mutually disjoint elements belonging to $\phi$, then $\left\{a_{n}-b\right\}$ for any $b \in C$ is a sequence of mutually disjoint elements belonging to $C$, hence $\bigcup_{n} a_{n}-b=\bigcup_{n}\left(a_{n}-b\right) \in C$. This means that $U_{n} a_{n} \in \phi$. But $\phi$ is closed under forming of complements too. In fact, let $a \in \phi$. We have $a-b \in C$ for any $b \in C$, so $\Omega-(a-b)$ $\in C$. Further $b \in C$ and $b \subset \Omega-(a-b)$, hence in view of (i) of the lemma $[\Omega-(a-b)]-b \in C$. Thus $(\Omega-a)-b=[\Omega-(a-b)]-b \in C$. The last gives $\Omega-a \in \phi$. So $\phi$ is a $\sigma$-class containing $A$, hence $\phi=C$. The validity $a-b \in C$ is proved for any $a, b \in C$. This completes the proof of (iii).

The sufficiency of (iv) follows from that of (iii). In fact, let $a, b \in A$. Under the assumption of (iv) $a \cap b \in C$. Since $a \cap b \subset a$, we have by (i) of the lemma $a-b=a-a \cap b \in C$. Thus (iii) is satisfied and the conclusion follows. 
In [1] the collection of sets is said to be compatible in $C$ if $a \cap b \in C$ for any $a, b \in A$. If $a \cap b \in A$ for any $a, b \in A$, then $A$ is said to be internally compatible.

In the above terminology (iv) of the theorem states that the $\sigma$-class generated by the collection $A$ is a $\sigma$-field if and only if $A$ is compatible in $C$. As a special case one gets the following

Corollary 1. If $A$ is internally compatible then the $\sigma$-class generated by $A$ is a $\sigma$-field.

This corollary generalises the Corollary 3.3 from [1]. Let us mention that there is a conjecture of the author of [1] stating that his Corollary 3.3 cannot be strengthened in the manner formulated above. Such a conjecture follows from the following slight error.

There is an assertion in [1], that the $\sigma$-class $C_{1}$ generated by the class

$$
A=\{a, b, c,\{1,2\},\{1,3\},\{1,6\},\{1\}\}
$$

where $a=\{1,2,3,4\}, b=\{1,2,5,6\}, c=\{1,3,6,8\}$ is contained in the $\sigma$-class consisting of all subsets of $\Omega=\{1,2, \cdots, 8\}$ with an even number of elements. This is evidently not true. Of course the mentioned error does not harm any of the results of the paper [1].

Applying Corollary 1 to the case of all open sets of a topological space one can get the following corollary giving the answer to one question appearing in [1].

Corollary 2. The Borel field (i.e. the $\sigma$-field generated by the collection of all open sets) in any topological space coincides with the $\sigma$-class generated by the collection of all open sets.

I am indebted to the referee for the Note and Corollary 3 which follow.

Note. The internal compatibility of $A$ is not necessary for the $\sigma$-class generated by $A$ to be a $\sigma$-field. For example, let $\Omega=\{1,2,3\}, A$ $=\{\{1,2\},\{2,3\}\}$.

Corollary 3. Let $(\Omega, C, M)$ be a quantum probability space and let $f: \Omega \rightarrow R$ be a real valued function. If $f^{-1}(\Lambda) \in C$ for every open set $\Lambda$ or even $f^{-1}((a, \infty)) \in C$ for every $a \in R$, then $f$ is observable (in the sense of $[1])$.

Proof. Let $S=\left\{\Lambda \subset R: f^{-1}(\Lambda) \in C\right\}$. Then $S$ is a $\sigma$-class containing $(a, \infty)$ for all $a \in R$. Since these intervals are internally compatible the $\sigma$-class generated by them is a $\sigma$-field which is in fact, $B(R)$. Thus $B(R) \subset S$. 


\section{REFERENCES}

1. S. P. Gudder, Quantum probability spaces, Proc. Amer. Math. Soc. 21 (1969), 296-302.

2. P. Suppes, The probabilistic argument for a non-classical logic of quantum mechanics, Philos. Sci. 33 (1966), 14-21. MR 35 \#6415.

3. W. Sierpinski, Les ensembles boreliens abstraits, Ann. Soc. Polon. Math. 6 (1927), 50-53.

4. P. R. Halmos, Measure theory, Van Nostrand, Princeton, N. J., 1950. MR 11, 504.

J. A. Komensky University, Bratislava, Czechoslovakia 